\title{
Lead poisoning due to appendiceal foreign body
}

\author{
Gustavo Stringel*, Camelia Lawrence, Richard Noto, Adele Brudnicki, Gwen Smith \\ Maria Fareri Children's Hospital/New York Medical College, Larchmont, USA \\ Email: *GStringel@aol.com
}

Received 24 June 2013; revised 23 July 2013; accepted 31 July 2013

Copyright (C 2013 Gustavo Stringel et al. This is an open access article distributed under the Creative Commons Attribution License, which permits unrestricted use, distribution, and reproduction in any medium, provided the original work is properly cited.

\begin{abstract}
Lead poisoning caused by a foreign body is rare. Foreign bodies that are ingested typically pass without consequence or awareness by the patient [1]. There are few documented cases of lead poisoning from an ingested foreign body lodged in the appendix. Screening for lead poisoning is mandated by New York State at annual well person exams for children ages 6 months to 6 years [2]. We present a case of a 2-yearold male who was found to have elevated lead levels during a routine well-child visit. An abdominal X-ray was obtained as part of routine follow up for elevated lead levels and a foreign body was found in the right lower quadrant. After unsuccessful attempt to flush the foreign body out with golytely, a CT abdomen/ pelvis was obtained. The foreign body was localized in the appendix. The patient was subsequently taken to surgery for an appendectomy to remove the foreign body. This case is a demonstration of the positive effects of the lead screening guidelines of New York.
\end{abstract}

Keywords: Lead Poisoning; Appendix Foreign Body

\section{CASE STUDY}

A 2-year-old boy presented to his pediatrician for an annual well-child examination. Lead levels were obtained as part of the routine blood screening for children. The lead level was reported to be 57 micrograms/dL (normal $<10$ micrograms/dL) leading to subsequent follow up. Although the patient was asymptomatic, he was admitted to the hospital for evaluation of lead toxicity and films were obtained. An abdominal X-ray revealed a metallic foreign body located in the right lower quadrant (Figure 1). Golytely was given in attempt to flush the foreign body out naturally. Abdominal films were repeated after the patient's bowels had been flushed. The films demonstrated the foreign body had not moved and a CT scan

${ }^{*}$ Corresponding author. was obtained to further localize the object (Figures 2(a) and (b)). Results showed the foreign body was most likely in the appendix without one hundred percent certainty due to artifact caused by the metallic object. A surgical consultation was requested and the patient was taken to the operating room for an appendectomy to remove the foreign body. The appendix was removed and an X-ray of the specimen was obtained to confirm that it contained the foreign body (Figure 3). A post-operative abdominal film confirmed there was no remaining metal (Figure 4). Pathology evaluated the foreign body which was then sent for spectroscopy to identify its chemical make up (Figures 5(a) and (b)). Pre-operatively the lead level reached 73 micrograms/dL. Chelation therapy was started post-operatively and the patient's lead levels trended down.

\section{DISCUSSION}

Childhood lead poisoning was first discovered in Australia in 1892 and then described in the United States in 1914 [3]. At that time it was believed that the children would either die from the lead toxicity or would live without any residual effects. In 1943 follow up of patients that survived lead poisoning demonstrated that there were residual effects in the form of learning disabilities and behavioral problems [3]. Asymptomatic children with lead poisoning and residual effects were discovered in the 1970s [3]. The threshold for lead toxicity was set at 60 micrograms/dL at that time [3]. This value has decreased in small increments to a level of 10 micrograms/dL which is the current statute written in 1993 for New York [2]. Lead toxicity values are expected to decrease again to 5 micrograms/dL or below since recent research has shown learning and behavioral deficits occurring at levels less than 5 micrograms/dL. [4].

Appendiceal foreign bodies are usually asymptomatic but can cause appendicitis or lead poisoning. There have been some cases in which adults and older children have presented with symptomatic lead poisoning caused by 


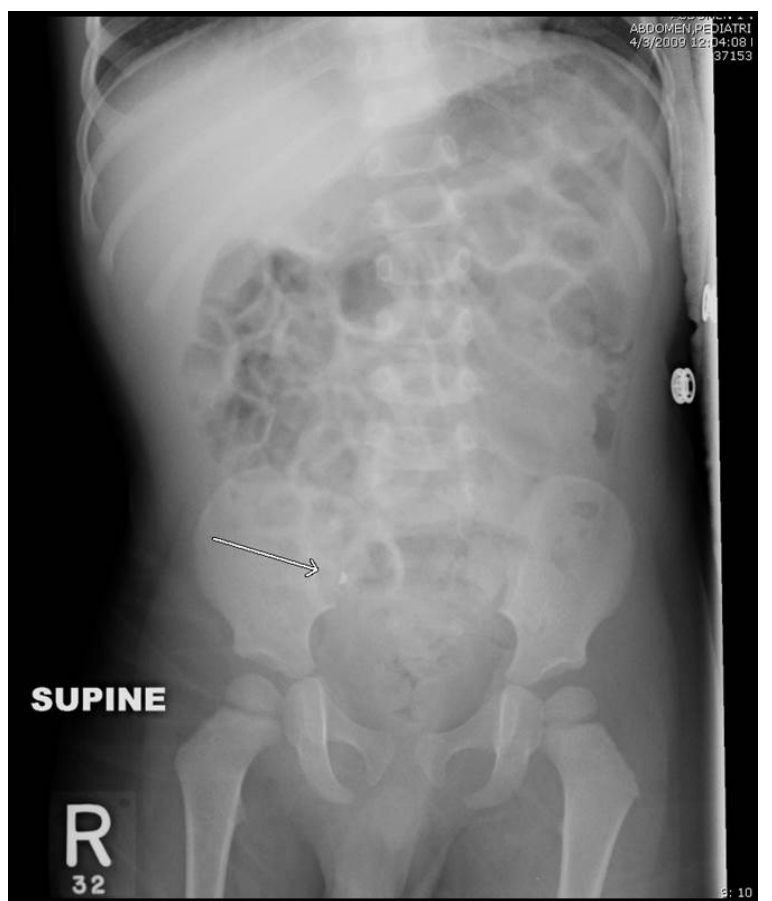

Figure 1. Abdominal radiograph demonstrating a metallic foreign body in the right lower quadrant.

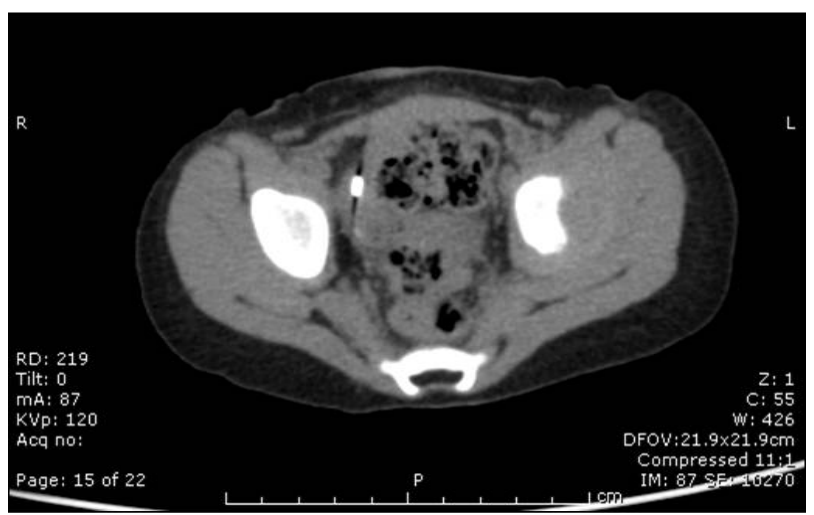

(a)

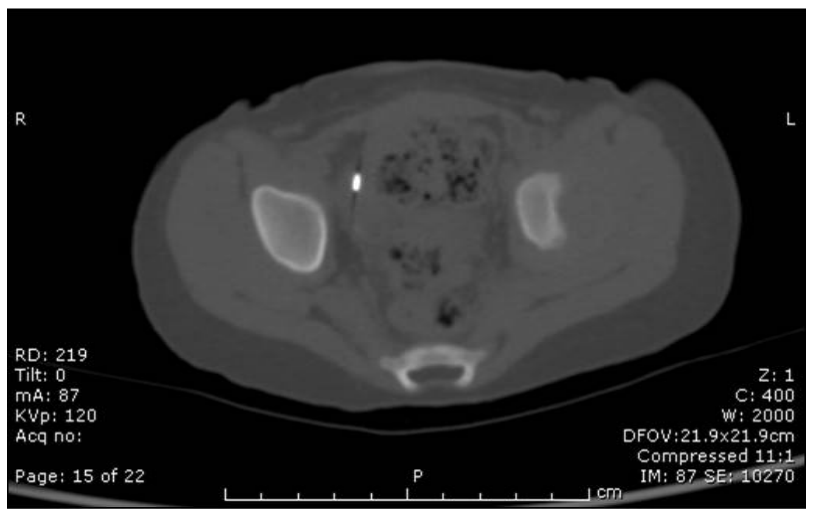

(b)

Figure 2. (a) and (b) Computerized Tomography of the abdomen (CT scan) demonstrating a foreign body in the right lower quadrant.

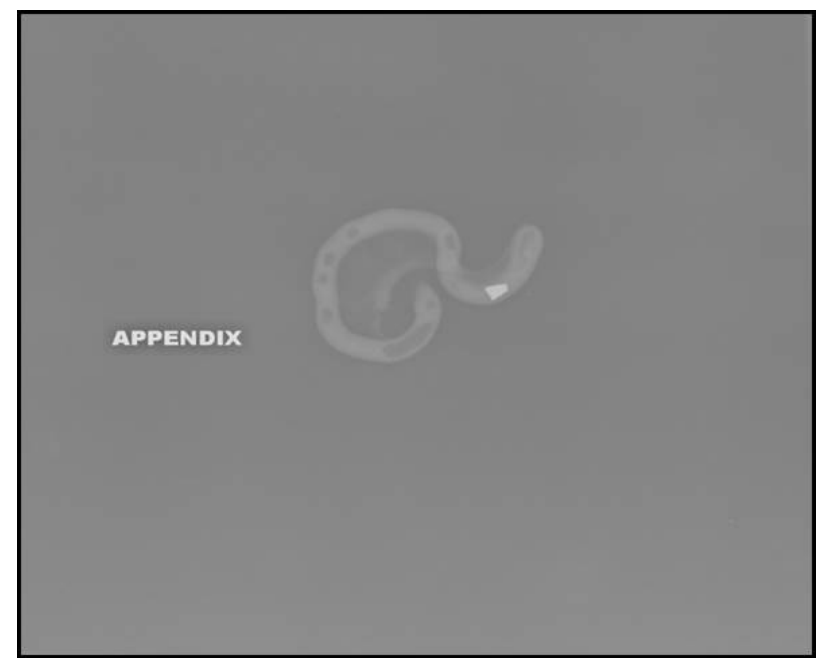

Figure 3. Radiograph of the appendix (specimen) containing an intraluminal metallic foreign body.

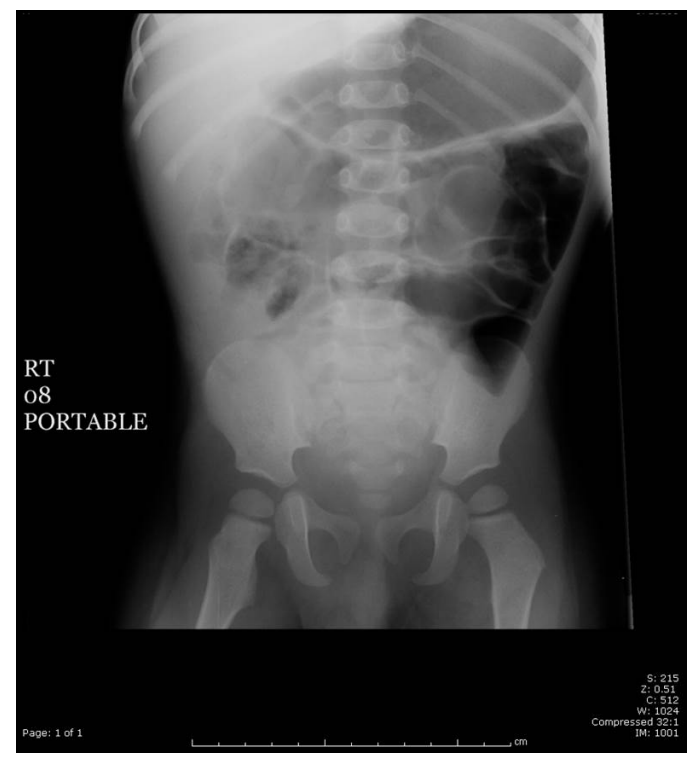

Figure 4. Postoperative radiograph after removal of the foreign body.

foreign bodies in the gastrointestinal tract [5]. The lead levels in these cases were significantly higher than our patient. Lead foreign bodies in the gastrointestinal tract of children can be more dangerous because children have a higher absorption rate of lead than adults at 30\% - 40\% versus 15\% - 20\%. [5] New York statute requires annual screening at well person examinations for children ages 6 months to 6 years of age [2].

\section{CONCLUSION}

Lead poisoning can be caused by foreign bodies, so follow up film for lead toxicity is useful in ruling out objects as the cause. Since foreign bodies pass through the body naturally, additional films should be taken to ob- 


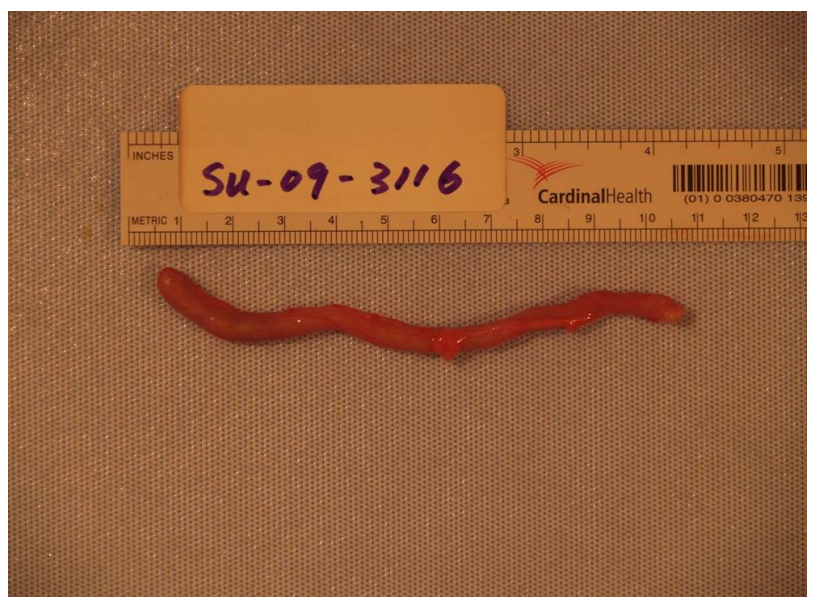

(a)

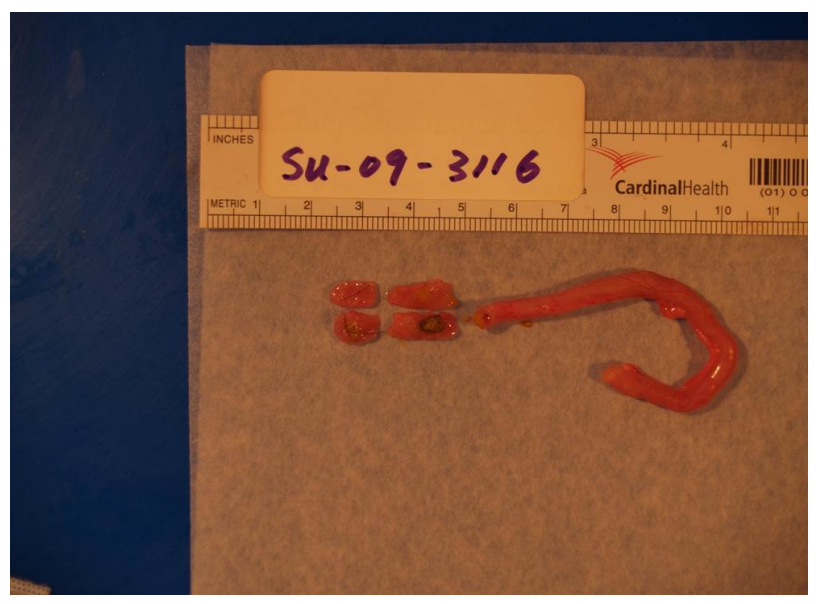

(b)

Figures 5. (a) and (b) Appendix containing the lead foreign body. serve for movement of object prior to seeking surgery. Children are at a higher risk for lead toxicity from ingestion of foreign bodies because they have a higher absorption rate of lead in their gastrointestinal tract. [5] The threshold for lead toxicity has been decreasing based on continuing evidence of residual effects from lower levels. [4].

\section{REFERENCES}

[1] Larsen, A.R. and Blanton, R.H. (2000) Appendicitis due to bird shot ingestion: A case study. The American Surgeon, 66, 589-591.

[2] Center for Disease Control (2009) Statutory authority: Public health law, section 206 and title X of article 13, subpart 67-1.

http://www.cdc.gov/nceh/lead/grants/New\%20York/new yorkstatescreen.pdf

[3] Needleman, H. (2004) lead poisoning. Annual Review of Medicine, 55, 209-222. doi:10.1146/annurev.med.55.091902.103653

[4] Kim, D., Overstreet Galeano, M.A., Hull, A. and Miranda, M.L. (2008) A framework for widespread replication of a highly spatially resolved childhood lead exposure risk model. Environmental Health Perspectives, 116, 17351739. doi:10.1289/ehp. 11540

[5] Gustavsson, P. and Gerhardsson, L. (2005) Intoxication from an accidentally ingested lead shot retained in the gastrointestinal tract. Environmental Health Perspectives, 113, 491-493. doi:10.1289/ehp.7594 